\title{
Transverse depinning and melting of a moving vortex lattice in driven periodic Josephson junction arrays
}

\author{
Verónica I. Marconi and Daniel Domínguez \\ Centro Atómico Bariloche, 8400 S. C. de Bariloche, Rio Negro, Argentina
}

(October 16, 2018)

\begin{abstract}
We study the effect of thermal fluctuations in a vortex lattice driven in the periodic pinning of a Josephson junction array. The phase diagram current $(I)$ vs. temperature $(T)$ is studied. Above the critical current $I_{c}(T)$ we find a moving vortex lattice (MVL) with anisotropic Bragg peaks. For large currents $I \gg I_{c}(T)$, there is a melting transition of the MVL at $T_{M}(I)$. When applying a small transverse current to the MVL, there is negligible dissipation at low $T$. We find an onset of transverse vortex motion at a transverse depinning temperature $T_{t r}(I)<T_{M}(I)$.
\end{abstract}

PACS numbers: 74.50+r, 74.60.Ge, 74.60.Ec

The interplay between the periodicity of vortex lattices (VL) and periodic pinning potentials in superconductors raises many interesting questions both in equilibrium [1] 6] and in driven systems [7]. Experimentally, periodic pinning has been realized in artificially fabricated systems like: superconducting wire networks [1], Josephson junction arrays [2, thickness modulated superconducting films [3], magnetic dot arrays [4] and submicron hole lattices [5] in superconductors. commensurability effects in the ground state vortex configurations lead to enhanced critical currents and resistance minima for "fractional" and for "matching" (i.e. commensurate) vortex densities where the VL is strongly pinned. Under the effect of thermal fluctuations, it is possible to have a depinning phase transition of these commensurate ground states at a temperature $T_{p}$ and a later melting transition of the VL at a temperature $T_{M}$ [6]. For high vortex densities (i.e. strongly interacting VL) both transitions coincide, $T_{p}=T_{M}$, while for low vortex densities both transitions are different with $T_{p}<T_{M}$. Out of equilibrium, many recent studies have concentrated in the related problem of the driven VL in the presence of random pinning 8 13. The nature of the fastly moving vortex structure for large driving forces has been under active discussion lately [8 10 motivating both experimental [11] and numerical [12 14] studies. In particular, the interesting concept of transverse critical current has been introduced in Ref. [9]. After applying a current in the direction perpendicular to the drive, a finite transverse critical current $I_{c, t r}$ may exist, at least at $T=0$ [9]. For $T>0$, there is a very small but finite transverse linear response [10], with a possible sharp non-linear voltage increase at an "effective" $I_{c, t r}$ 9, 10]. For periodic pinning, the physics of the driven VL has been studied numerically only at $T=0$, where a complex variety of dynamic phases has been reported [7. In this case, it is clear that a finite $I_{c, t r}$ will exist due to commensurability effects [10], and it has been obtained in [7] for $T=0$.

In this Letter we study the effect of thermal fluctuations in a driven VL in a periodic pinning potential. In our case, the periodic pinning is provided by a two di- mensional Josephson junction array (JJA) [2, 15, 16]. We obtain a phase diagram as a function of the driving current $(I)$ and temperature $(T)$, which is shown in Fig. 1. For low currents, we find that the depinning and melting transitions are separated with $T_{p}(I)<T_{M}(I)$. More interestingly, for large currents we find an analogous sequence of transitions but for the transverse response of a fastly moving VL. We find that there is a novel transverse depinning temperature $T_{t r}$ below the melting transition of the moving VL, $T_{t r}(I)<T_{M}(I)$.

The current flowing in the junction between two superconducting islands in a JJA is modeled as the sum of the Josephson supercurrent and the normal current [15,16]:

$$
I_{\mu}(\mathbf{n})=I_{0} \sin \theta_{\mu}(\mathbf{n})+\frac{\Phi_{0}}{2 \pi c R_{N}} \frac{\partial \theta_{\mu}(\mathbf{n})}{\partial t}+\eta_{\mu}(\mathbf{n}, t)
$$

where $I_{0}$ is the critical current of the junction between the sites $\mathbf{n}$ and $\mathbf{n}+\mu$ in a square lattice $\left[\mathbf{n}=\left(n_{x}, n_{y}\right)\right.$, $\mu=\hat{\mathbf{x}}, \hat{\mathbf{y}}], R_{N}$ is the normal state resistance and $\theta_{\mu}(\mathbf{n})=$ $\theta(\mathbf{n}+\mu)-\theta(\mathbf{n})-A_{\mu}(\mathbf{n})=\Delta_{\mu} \theta(\mathbf{n})-A_{\mu}(\mathbf{n})$ is the gauge invariant phase difference with $A_{\mu}(\mathbf{n})=\frac{2 \pi}{\Phi_{0}} \int_{\mathbf{n} a}^{(\mathbf{n}+\mu) a} \mathbf{A}$. $d \mathbf{l}$. The thermal noise fluctuations $\eta_{\mu}$ have correlations $\left\langle\eta_{\mu}(\mathbf{n}, t) \eta_{\mu^{\prime}}\left(\mathbf{n}^{\prime}, t^{\prime}\right)\right\rangle=\frac{2 k T}{R_{N}} \delta_{\mu, \mu^{\prime}} \delta_{\mathbf{n}, \mathbf{n}^{\prime}} \delta\left(t-t^{\prime}\right)$. In the presence of an external magnetic field $H$ we have $\Delta_{\mu} \times$ $A_{\mu}(\mathbf{n})=A_{x}(\mathbf{n})-A_{x}(\mathbf{n}+\mathbf{y})+A_{y}(\mathbf{n}+\mathbf{x})-A_{y}(\mathbf{n})=2 \pi f$, $f=H a^{2} / \Phi_{0}$ and $a$ is the array lattice spacing. We take periodic boundary conditions (p.b.c) in both directions in the presence of an external current $I_{\text {ext }}$ in the $y$-direction in arrays with $L \times L$ junctions [14. The vector potential is taken as $A_{\mu}(\mathbf{n}, t)=A_{\mu}^{0}(\mathbf{n})-\alpha_{\mu}(t)$ where in the Landau gauge $A_{x}^{0}(\mathbf{n})=-2 \pi f n_{y}, A_{y}^{0}(\mathbf{n})=0$ and $\alpha_{\mu}(t)$ will allow for total voltage fluctuations. With this gauge the p.b.c. for the phases are: $\theta\left(n_{x}+L, n_{y}\right)=\theta\left(n_{x}, n_{y}\right)$ and $\theta\left(n_{x}, n_{y}+L\right)=\theta\left(n_{x}, n_{y}\right)-2 \pi f L n_{x}$. The condition of a current flowing in the $y$ - direction: $\sum_{\mathbf{n}} I_{\mu}(\mathbf{n})=$ $I_{\text {ext }} L^{2} \delta_{\mu, y}$ determines the dynamics of $\alpha_{\mu}(t)$ [14]. After considering conservation of current, $\Delta_{\mu} \cdot I_{\mu}(\mathbf{n})=$ $\sum_{\mu} I_{\mu}(\mathbf{n})-I_{\mu}(\mathbf{n}-\mu)=0$, we obtain:

$$
\Delta_{\mu}^{2} \frac{\partial \theta(\mathbf{n})}{\partial t}=-\Delta_{\mu} \cdot\left[S_{\mu}(\mathbf{n})+\eta_{\mu}(\mathbf{n}, t)\right]
$$




$$
\frac{\partial \alpha_{\mu}}{\partial t}=I_{e x t} \delta_{\mu, y}-\frac{1}{L^{2}} \sum_{\mathbf{n}}\left[S_{\mu}(\mathbf{n})+\eta_{\mu}(\mathbf{n}, t)\right]
$$

where $S_{\mu}(\mathbf{n})=\sin \left[\Delta_{\mu} \theta(\mathbf{n})-A_{\mu}^{0}(\mathbf{n})-\alpha_{\mu}\right]$, we have normalized currents by $I_{0}$, time by $\tau_{J}=2 \pi c R_{N} I_{0} / \Phi_{0}$, temperature by $I_{0} \Phi_{0} / 2 \pi k_{B}$, and the discrete laplacian is $\Delta_{\mu}^{2} \theta(\mathbf{n})=\theta(\mathbf{n}+\hat{\mathbf{x}})+\theta(\mathbf{n}-\hat{\mathbf{x}})+\theta(\mathbf{n}+\hat{\mathbf{y}})+\theta(\mathbf{n}-\hat{\mathbf{y}})-4 \theta(\mathbf{n})$.

The Langevin dynamical equations (2-3) are solved with a second order Runge-Kutta-Helfand-Greenside algorithm with time step $\Delta t=0.1 \tau_{J}$ and integration time $10000 \tau_{J}$ after a transient of $5000 \tau_{J}$. The discrete laplacian is inverted with a fast Fourier + tridiagonalization algorithm as in [16]. We study the following properties: (i) Superconducting coherence: we calculate the helicity modulus in the direction transverse to the current $\Upsilon_{x}=\frac{1}{L^{2}}\left\langle\sum_{\mathbf{n}} \cos \theta_{x}(\mathbf{n})\right\rangle-\frac{1}{T} \frac{1}{L^{4}}\left\{\left\langle\left[\sum_{\mathbf{n}} \sin \theta_{x}(\mathbf{n})\right]^{2}\right\rangle-\right.$ $\left.\left\langle\left[\sum_{\mathbf{n}} \sin \theta_{x}(\mathbf{n})\right]\right\rangle^{2}\right\}$. [In order to calculate the helicity modulus along $x$, we enforce strict periodicity in $\theta$ by fixing $\alpha_{x}(t)=0$ ]. (ii) Transport: we calculate the time average of the total voltage $V=\left\langle v_{y}(t)\right\rangle=\left\langle d \alpha_{y}(t) / d t\right\rangle$ (voltages are normalized by $R_{N} I_{0}$ ). (iii) Vortex structure: we obtain the vorticity at the plaquette $\tilde{\mathbf{n}}$ (associated to the site $\mathbf{n})$ as $b(\tilde{\mathbf{n}})=-\Delta_{\mu} \times \operatorname{nint}\left[\theta_{\mu}(\mathbf{n}) / 2 \pi\right]$ with $\operatorname{nint}[x]$ the nearest integer of $x$. We calculate the average vortex structure factor as $S(\mathbf{k})=\left\langle\left|\frac{1}{L^{2}} \sum_{\tilde{\mathbf{n}}} b(\tilde{\mathbf{n}}) \exp (i \mathbf{k} \cdot \tilde{\mathbf{n}})\right|^{2}\right\rangle$.

We study JJA with a magnetic field corresponding to $f=1 / 25$ and system sizes of $L \times L$ junctions, with $L=50,100$. The ground state vortex configuration for $f=1 / 25$ is a tilted square-like vortex lattice (VL) commensurate with the underlying periodic pinning potential of the square JJA (see [17]). The structure factor $S(\mathbf{k})$ has correspondingly delta-like Bragg peaks. For this value of $f$ we find an equilibrium phase transition at $T_{c} \approx 0.050 \pm 0.003$, which corresponds to a simultaneous VL depinning (corresponding to the onset of resistivity and vanishing of helicity modulus) and VL melting (corresponding to the vanishing of Bragg peaks); i.e. $T_{c}=T_{p}=T_{M}$.

First, we have calculated the current-voltage (IV) characteristics for different temperatures. At $T=0$ there is a critical current of $I_{c}(0)=0.114 \pm 0.002$, which corresponds to the single vortex depinning current in square JJA [18]. Above $I_{c}(0)$ there is an almost linear increase of voltage until $I \approx 1$ where there is a sharp rise of $V$ because all the junctions become normal. Similar behavior has been reported for $T=0$ IV curves for low values of $f$ [19]. We restrict our analysis for currents $I<0.4$, where the collective behavior of the VL is the dominant physics. For temperatures $T<T_{c}$ we see that there is a sharp rise in voltage for the apparent critical current $I_{c}(T)$, which decreases with $T$, vanishing at $T_{c}$. In Fig. 1 we plot the $I_{c}(T)$ line obtained with a voltage criterion of $V<10^{-4}$. For currents below $I_{c}(T)$ there is a pinned vortex lattice (PVL) which is the same as the $T=0$ ground state, with delta-like Bragg peaks. On the other hand, for currents $I>I_{c}(T)$ there is a moving vortex lattice (MVL), which has anisotropic Bragg peaks in the structure factor $S(\mathbf{k})$ as shown in Fig. 2(a). There are two features in the anisotropy of $S(\mathbf{k})$ : (i) The height of the peaks decreases in the direction of vortex motion (i.e. perpendicular to the current drive). (ii) The width of the peaks increases in the direction perpendicular to vortex motion. This means that thermal broadening is less effective in the direction of motion. We have also studied the behavior of the Bragg peaks of the PVL and the MVL for three different lattice sizes $(L=50,100,150)$ and different values of $I$ and $T$. In the PVL the peak height is independent of the system size, as expected for a pinned lattice. On the other hand, for the MVL, the peak height decreases with system size, with a power law behavior $S(\mathbf{G}) \sim L^{-\eta_{G}(I, T)}$ and $0<\eta_{G}(I, T)<2$. In Fig. 2(b) we show a finite size analysis for two Bragg peaks $S\left(G_{1}\right)$ and $S\left(G_{2}\right)$ at a particular point in $(I, T)$ with a power law fit. This is the expected behavior for a floating solid in two dimensions [6]. In general we see that $\eta_{G}$ increases with $T$ for a given current. When $\eta_{G}(I, T)>2$ the MVL melts into a liquid. The anisotropic structure of the MVL of Fig. 2(a) is similar to the behavior predicted for a moving Bragg glass [9]. However in our case there is no random pinning, but periodic pinning. The only source of dynamic randomness are thermal fluctuations.

We now study in more detail the different transitions by fixing a given value of the current $I$ and slowly changing temperature $T$ with small increases of $\Delta T=0.0005$. In this way we obtain the phase diagram shown in Fig. 1. We have also cross-checked these results with the IV curves at fixed $T$. There are two cases of interest: (i) low currents $I<I_{c}(0)$ and (ii) large currents $I>I_{c}(0)$.

(i)Low currents. In Fig. 3 we show the behavior for $I=0.03<I_{c}(0)$. At low temperatures the voltage is almost zero since the VL is pinned. When increasing $T$ there is a sharp rise of the voltage (of two orders of magnitude) at a depinning temperature $T_{p}(I)$, as shown in Fig.3(a). At this temperature the VL starts to move since the driving current is higher than the critical current. Therefore, this corresponds to a transition from a pinned VL to a moving VL. We find that the $I_{c}(T)$ line obtained from the IV curves at fixed $T$ coincides with the $T_{p}(I)$ line obtained from the $V-T$ curves. We have also calculated at the same time the structure factor $S(\mathbf{k}, T)$ and the transverse helicity modulus $\Upsilon_{x}(T)$. In Fig. 3(b) we show the behavior of two Bragg peaks $S\left(G_{1}\right)$ and $S\left(G_{2}\right)$. For $T<T_{p}(I)$ we see that $S\left(G_{1}\right)=S\left(G_{2}\right)$ since there is a pinned VL with isotropic structure factor. Above $T_{p}(I)$ we find that $S\left(G_{1}\right) \neq S\left(G_{2}\right)$. This shows the fact that there is a MVL with anisotropic Bragg peaks. These peaks vanish at a higher temperature $T_{M}(I)$ in a continuous and smooth transition, corresponding to a melting of the MVL. Above $T_{M}(I)$ all Bragg peaks vanish and there is a vortex liquid for $T>T_{M}$. In the inset of Fig. 3(b) we show $S\left(G_{1}\right)$ for two different sizes $L=50,100$ [we find similar finite size effects for $\left.S\left(G_{2}\right)\right]$. We see that for 
$T<T_{p}(I)$ the $S\left(G_{1}\right)$ is size independent since the $\mathrm{VL}$ is pinned, while for $T_{p}(I)<T<T_{M}(I)$ there is a powerlaw size dependence as expected for a floating solid, see Fig. 2(b). The temperature $T_{M}(I)$ where $S\left(G_{1}\right)$ vanishes is size independent. On the other hand, the $\Upsilon_{x}(T)$ has a significant drop at $T_{p}(I)$, however it remains finite but with large fluctuations in the MVL phase, $T_{p}<T<T_{M}$. This suggests that in the MVL there is superconducting coherence in the direction transverse to the current.

(ii) Large currents. When the VL is driven with a large current $I>I_{c}(0)$ there is a moving VL with anisotropic Bragg peaks even at $T=0$. In Fig. 4 we show our results for $I=0.16>I_{c}(0)$. The structure factor is always anisotropic as can be seen in Fig. 4(b) where $S\left(G_{1}\right) \neq S\left(G_{2}\right)$. We find that the Bragg peaks vanish at a temperature $T_{M}(I)$, which is size independent, as shown in the inset of Fig. 4(b) for $S\left(G_{1}\right)$ [similar behavior is found for $S\left(G_{2}\right)$ ]. We have investigated the possibility of a transverse critical current $I_{c, t r}$ [9,10]. At $T=0$ a finite $I_{c, t r}$ is expected due to commensurability effects 10, ]. We have applied a transverse current $I_{t r}=I_{x}$ in the $x$-direction (in addition to the applied bias, $\left.I=I_{y}=0.16\right)$ and we have calculated the transverse voltage response $V_{t r}$. We find that for finite low temperatures $V_{t r}$ is negligible small within our numerical accuracy, until there is a sharp increase at an "effective" transverse critical current $I_{c, t r}(T)$. We find that $I_{c, t r}(T)$ tends to vanish at a temperature $T_{t r}$. An interesting way of studying this phenomenon is to apply a small current $I_{t r}$ and vary $T$. In Fig. 4(a) we study the onset of the transverse depinning transition: we apply a small current $I_{t r}=0.01$, and we show the transverse resistive response $V_{t r} / I_{t r}$ vs $T$. We see that $V_{t r}$ is vanishingly small at low $T$ and it rises at $T_{t r}$. This transition temperature is clearly below $T_{M}$ as we can see in Fig. 4. We have obtained $T_{t r}(I)$ for two driving currents $I>I_{c}(0)$ as we show in Fig. 1. It seems reasonable that this transverse depinning line will exist all along this region of the phase diagram. For the intermediate temperatures $T_{t r}<T<T_{M}$ we find that there is always an ordered vortex array but the orientation and structure of the MVL depends on the initial conditions. Moreover, finite size analysis shows that for $T<T_{t r}$ the MVL structure factor is weakly size dependent with $\eta_{G}(T) \sim 0.05$, while after $T_{t r}$, the exponent $\eta_{G}$ has a steep increase to values $\eta_{G}(T) \sim 0.5$. The helicity modulus is shown in Fig. 4(c). We see that for $T<T_{t r}$ the MVL has transverse superconducting coherence with a well-defined $\Upsilon_{x}$. On the other hand, $\Upsilon_{x}$ shows strong fluctuations in the region $T_{t r}<T<T_{M}$.

In conclusion, we have studied the current-temperature phase diagram of a vortex lattice driven in a periodic Josephson junction array. We find that for low currents the "longitudinal" depinning transition of the VL and the later melting of the moving $\mathrm{VL}$ are different with $T_{M}(I)>T_{p}(I)$. For large currents we find a very analogous behavior for the "transverse" response of a fastly moving VL (compare Fig. 3 with Fig. 4). In this case it is possible to define a transverse depinning transition at a temperature $T_{t r}$, and a later melting transition of the moving VL at $T_{M}(I)$. This transverse depinning transition could be easily studied in controlled experiments in Josephson junction arrays with transport measurements.

We acknowledge Hernan Pastoriza for useful discussions and Fundación Antorchas and Conicet (Argentina) for financial support.

[1] B. Pannetier et al, Phys. Rev. Lett. 53, 1845 (1984).

[2] See for example M. S. Rzchowski et al, Phys. Rev. B 42, 2041 (1990). For a review see Macroscopic Quantum Phenomena and Coherence in Superconducting Networks, Ed. C. Giovanella and M. Tinkham (World Scientific, Singapore, 1995).

[3] P. Martinoli, Phys. Rev. B 17, 1175 (1978) .

[4] J. I. Martin et al., Phys. Rev. Lett. 79, 1929 (1997).

[5] V. V. Moshchalkov et al., Phys. Rev. B 57, 3615 (1998).

[6] M. Franz and S. Teitel, Phys. Rev. Lett. 73, 480 (1994) and Phys. Rev. B 51, 6551 (1995); S. Hattel and J. M. Wheatley, Phys. Rev. B 51, 11951 (1995).

[7] C. Reichhardt, C. J. Olson and F. Nori, Phys. Rev. Lett. 78, 2648 (1997); Phys. Rev. B 58, 6534 (1998).

[8] A. E. Koshelev and V. M. Vinokur, Phys. Rev. Lett. 73, 3580 (1994); S. Scheidl and V. M. Vinokur, Phys. Rev. B 57, 13800 (1998).

[9] T. Giamarchi and P. Le Doussal, Phys. Rev. Lett. 76, 3408 (1996); P. Le Doussal and T. Giamarchi, Phys. Rev. B 57, 11356 (1998).

[10] L. Balents, M. C. Marchetti and L. Radzihovsky, Phys. Rev. B 57, 7705 (1998).

[11] F. Pardo et al, Phys. Rev. Lett. 78, 4633 (1997); and Nature, to be published.

[12] K. Moon et al., Phys. Rev. Lett. 77, 2778 (1996); S. Ryu et al., Phys. Rev. Lett. 77, 5114 (1996); S. Spencer and H. J. Jensen, Phys. Rev. B 55, 8473 (1997); C. J. Olson et al., Phys. Rev. Lett. 81, 3757 (1998).

[13] D. Domínguez, N. Grønbech-Jensen and A.R. Bishop, Phys. Rev. Lett. 78, 2644 (1997).

[14] D. Domínguez, Phys. Rev. Lett. 82, 181 (1999).

[15] J. S. Chung, K. H. Lee, and D. Stroud, Phys. Rev. B 40, 6570 (1989); F. Falo et al., Phys. Rev. B 41, 10983 (1990); N. Grønbech-Jensen et al., Phys. Rev. B 46, 11149 (1992), P.H.E. Tiesinga et al., Phys. Rev. Lett. 798, 519 (1997).

[16] D. Domínguez et al., Phys. Rev. Lett. (1991) 67, 2367; D. Domínguez, Phys. Rev. Lett. 72, 3096 (1994).

[17] Y. H. Li and S. Teitel, Phys. Rev. B 47, 359 (1993).

[18] C. J. Lobb, D. W. Abraham. and M. Tinkham, Phys. Rev. B 27, 150 (1983).

[19] W. Yu, K. H. Lee, and D. Stroud, Phys. Rev. B 47, 5906 (1993); T. J. Hagenaars et al, Phys. Rev. B 50, 1143 (1994). 
FIG. 1. $\quad I-T$ Phase diagram for $f=1 / 25$. $T_{M}(I)$ line is obtained from $\Upsilon_{x}$ vs. $T$ curves $(\square)$ and from $S(\mathbf{G})$ vs. $T$ curves $(\bullet) . T_{p}(I)$ line is obtained from IV curves $(\star)$, from $S(\mathbf{G})$ vs. $T$ curves $(\bullet)$ and from $\left\langle V_{y}\right\rangle$ vs. $T$ curves $(\triangle) . T_{t r}(I)$ curve is obtained from $\left\langle V_{t r}\right\rangle$ vs. $T$ curves (o). Temperature is normalized by $I_{0} \Phi_{0} / 2 \pi k_{B}$.

FIG. 2. (a) Intensity plot of the structure factor $S(\mathbf{G})$ for a moving vortex lattice at $I=0.06, T=0.02$. (b) Finite size analysis and power law fit of $S(\mathbf{G}) \sim L^{-\eta_{G}}$; we obtain $\eta_{G_{1}}=0.25 \pm 0.06(*)$ and $\eta_{G_{2}}=0.14 \pm 0.05(\triangle)$.

FIG. 3. For $I<I_{c}(0), I=0.03$ : (a) Dissipation $\left\langle V_{y}\right\rangle / I$ vs. T. (b) Structure factor at two lattice vectors, $S\left(\mathbf{G}_{\mathbf{1}}\right)(\star)$ and $S\left(\mathbf{G}_{\mathbf{2}}\right)(\diamond)$ vs. $T$. Inset: Size effect in $S(\mathbf{G})$. (c) Helicity modulus $\Upsilon_{x}$ vs. $T$.

FIG. 4. For $I>I_{c}(0), I=0.16$ : (a) Transverse dissipation $\left\langle V_{t r}\right\rangle / I_{t r}$ vs. $T$. (b) $S\left(\mathbf{G}_{\mathbf{1}}\right)(\star)$ and $S\left(\mathbf{G}_{\mathbf{2}}\right)(\diamond)$ vs. T. Inset: Size effect in $S(\mathbf{G})$. (c) $\Upsilon_{x}$ vs. $T$. 


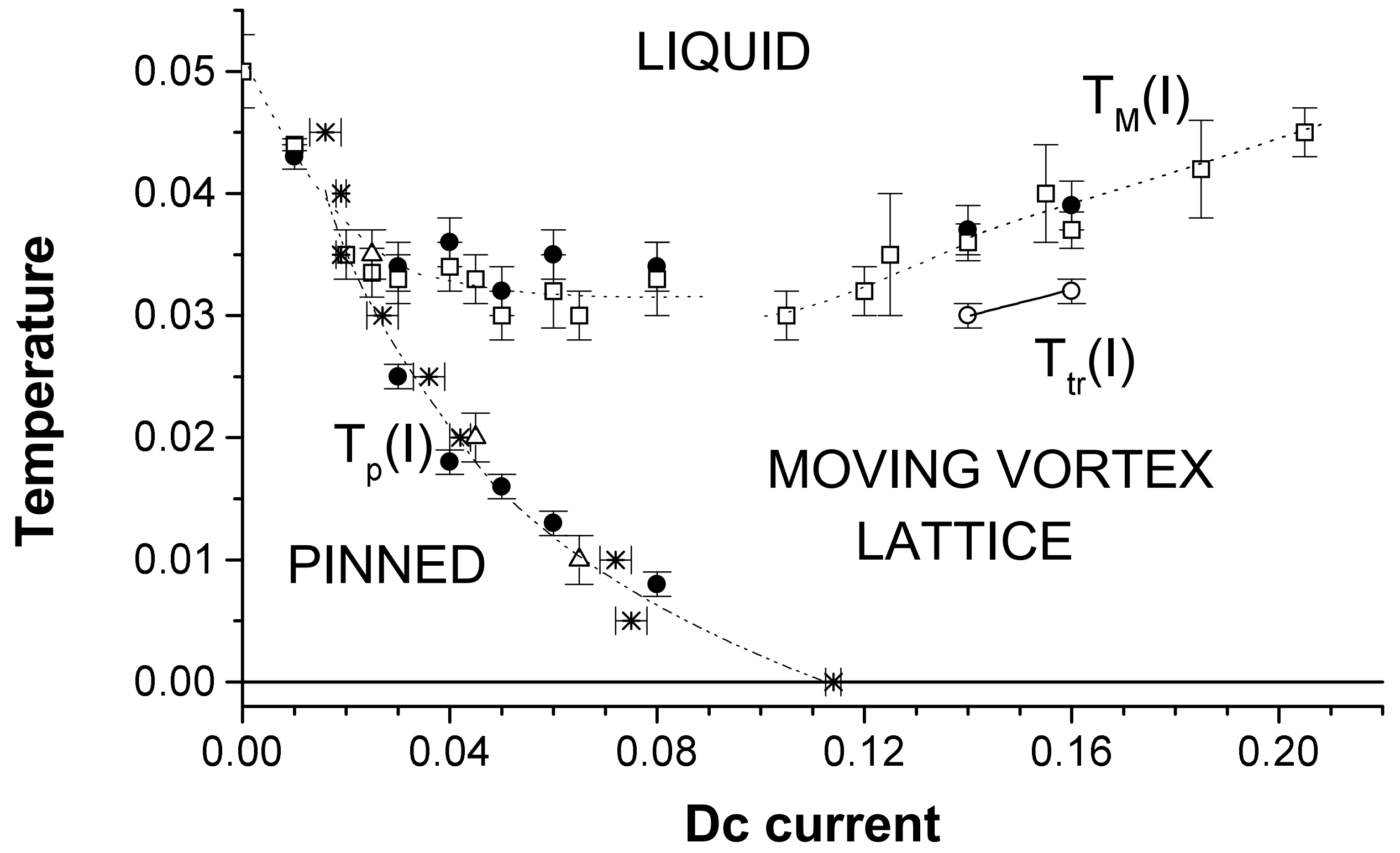



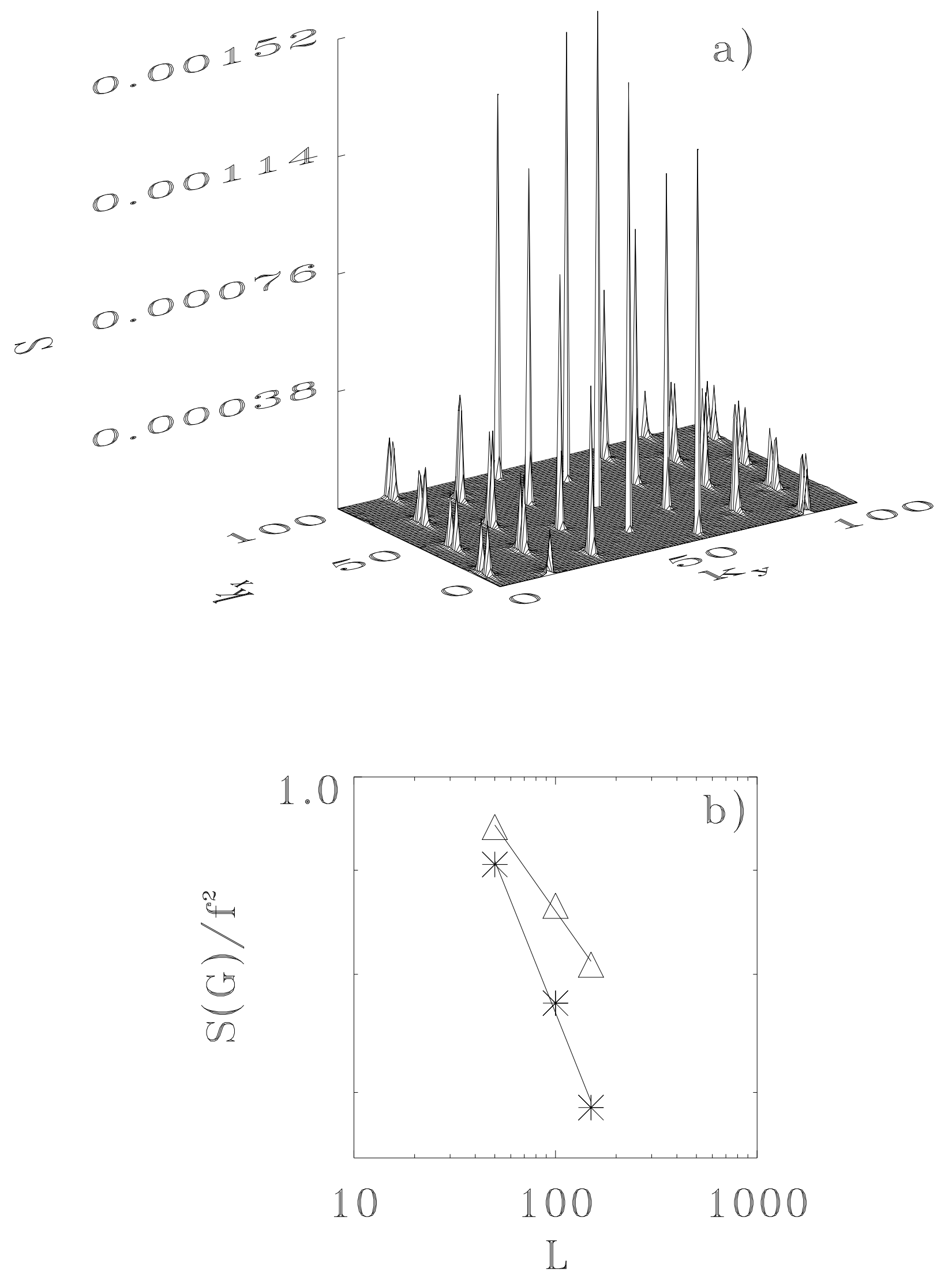


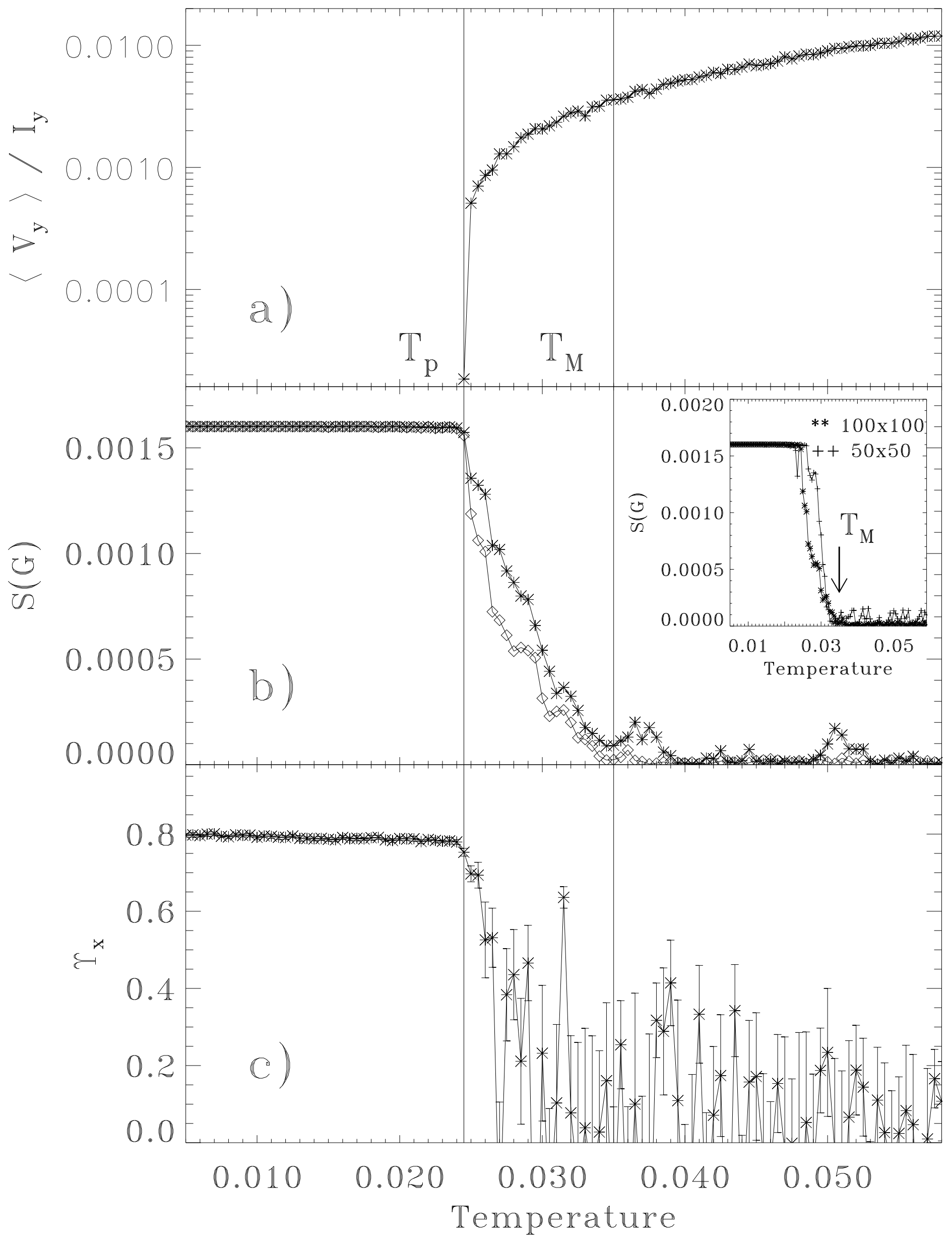




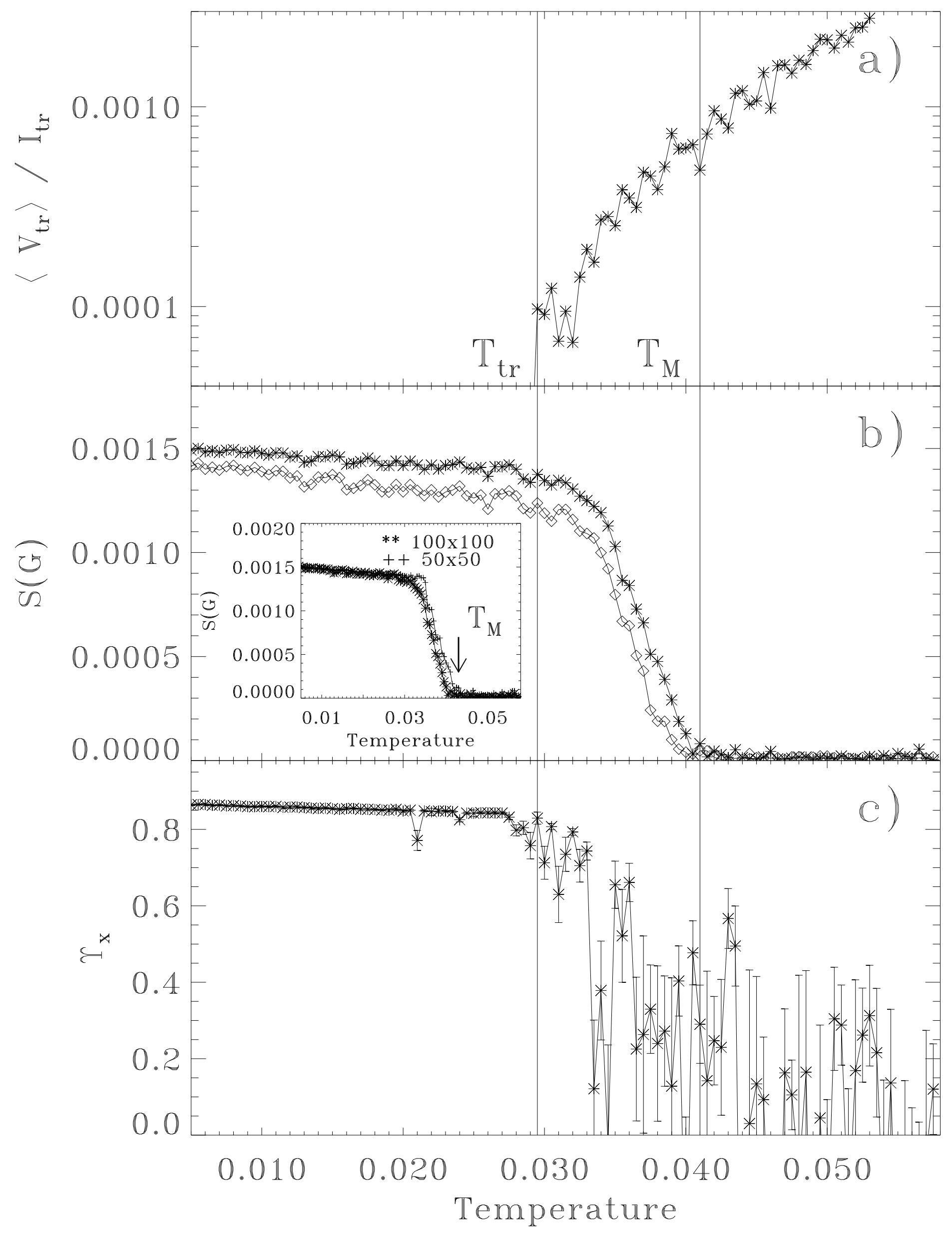

